\title{
Urgences
}

\section{La mémoire sombre}

\section{Constance Asplanato}

Numéro 33, octobre 1991

Poésies parallèles : France - Québec

URI : https://id.erudit.org/iderudit/025657ar

DOI : https://doi.org/10.7202/025657ar

Aller au sommaire du numéro

Éditeur(s)

Urgences

ISSN

0226-9554 (imprimé)

1927-3924 (numérique)

Découvrir la revue

Citer ce document

Asplanato, C. (1991). La mémoire sombre. Urgences, (33).

https://doi.org/10.7202/025657ar

Ce document est protégé par la loi sur le droit d'auteur. L'utilisation des services d'Érudit (y compris la reproduction) est assujettie à sa politique d'utilisation que vous pouvez consulter en ligne.

https://apropos.erudit.org/fr/usagers/politique-dutilisation/
Cet article est diffusé et préservé par Érudit.

Érudit est un consortium interuniversitaire sans but lucratif composé de l’Université de Montréal, l'Université Laval et l'Université du Québec à Montréal. Il a pour mission la promotion et la valorisation de la recherche. https://www.erudit.org/fr/ 
Constance Asplanato

Geneviève Amyot 


\section{La mémoire sombre}

\section{Constance Asplanato}

une fois le fleuve franchi, cet espace, ce presque vide, une étendue qui n'est et pourtant s'ouvre, se livre beaucoup moins qu'un livre mais, de force, de toute nécessité se forgeant, se faisant, - ou ce faisant -

se force ici et tente de se projeter se collecter, en ce silence ramassé

seule alternative existante, savoir que c'est toujours, surtout, se taisant -

oscillant entre deux pôles cherchés conciliables, contre toute, atteinte, interdite - dans cet intervalle non, encore, mesuré -

les deux pôles du dire, se faisant et se taisant par là, toujours hasardé, rêvé qui coïncide, et puis l'outrage

ici il cita la phrase, à ce lieu même du dire, la phrase attribuée à Rousseau, " ce pays de forgerons *, ce qu'invoquer la force est, de toute allusion faire acte à ce qui se forme et se modèle, rêvant et d'abolir et d'oublier des modèles passés

ce qui signifie encore une fois, et à son insu, les tenir pour existant, nécessitant de les ôter

se modéra, trouva dans le dialogue ou le crut cette façon même de se faire indifférente, trembla lorsqu'elle évoqua Giono et surtout le nom de ce hameau, le sien propre et pas seulement de Giono, le Contadour, et put se faire croire qu'il s'agissait un peu du froid, quand ce n'était que du soi, et parla de Michel aussi - pas le même et pourtant même ou de même tout intérieur -

c'est-à-dire tout aussi ôté, arraché à soi, et froid dorénavant dans la zone où il avait pris corps, sans et que ce dépourvu, ce détachement peutêtre souhaité, peut-être souhaitable, qui peut savoir, laisse sans,

sans voie, sans foi, sans peine ni repos avec cette seule haine pour peine légère (c'est-à-dire pour condamnation, pour être, prononcée) 
le nommé Michel, initial, perdu lui aussi, avait cédé bien vite, dans la dérive de la désignation, à une zone analogue et nommée,

cette toute façon, et de favoriser qui surgit, et de le brider ensuite au nom de qui établi,

rêvant or, et hors, de rejoindre les modèles passés de s'y, de les, et de la - aliéner, la distance entre l'autre et soi, que l'on abolit telle, que l'on abolit être, lorsqu'on sait à quel point ce profil même, le sien, aigu, désiré, est possédé et savouré au sein seul même de son image comportée, à travers cette fenêtre aujourd'hui ou qu'importe puisque suit

puisque suis

de se les aliéner, de vous aliéner à moi autre, que vous êtes, sans attenter à votre qualité d'étranger à moi, d'étranger à tout

cette puissance qu'est, votre distance ce concentré subit des rares rencontres soudaineté douce, peu après ôtée, pour, être et désirée - à de si rares accès Une fois ce fut dit, le fleuve franchi.

ce pouvait être quelque chose comme un effet de texte immédiat, comme naît, et ainsi s'évertue, à autre chose vite, que sa naissance réglée, cette facilité accordée, et par l'abstrait est dénié ici - mais il reste à être moins gratuit,

à savoir ce qu'est tout aussi superflu d'exister, puisque,:

cela me revient tout aussi soudainement, est-ce donc d'avoir parlé, ce Giono - puisque donc ce chemin depuis le haut, la maison, j'y ai vécu, à flanc de côteau, puisque donc le chemin, la mémoire y supplée ici mais sait bien les sensations qu'elle s'y recrée, qui passait par les touffes de thym, le chemin qui passait par la terrasse des manoscains, le chemin donc par les ruches et le haut - descendait à flanc d'églantier, s'y arrangeait pour perdre dix fois son monde mais aboutissait sur la route de terre battue, ravalée par les pluies, dans un virage où se postait un fragment de charrue, un peu élargi le virage et remblayé, et de là on pouvait juste franchir la route et se perdre en bas, se perdre juste comme il le fallait, juste en dédaignant la voie tracée et la traversant, vers l'endroit où un très vieux poirier avait donné parfois des fruits, et l'ombre 
10

redoutable d'un gros noyer, les tiges un peu sèches déjà des inflorescences, des camomilles, qui ne demandaient qu'à faire bouquet peut-être, et un peu âcre

oubliant elles-mêmes au fil des jours leur couleur, juste blonde, sur la raideur de leur tige, s'efface

même lors en pensée de longtemps n'y être retournée

est-ce, de taire ou de dire, ce genre de passé, qui donne la plus complète impression de l'avoir perdu - la mémoire n'était par toujours l'état de reflet, mais parfois l'état de rejet, ce mouvement qui expulsait hors de l'ombre où se tenaient, ou se perdaient, de tels déroulements et de tels chemins

- parfois on oublie juste qu'on est déjà mort -

les si nombreux réseaux inemployés, volontairement interdits il ne manquerait que d'en parler - de la mémoire, désaffection

et silence.

peut-être était-ce moins la mémoire comme instance dépositaire que la conscience tout simplement, qui s'est forgée de cela, qui ne les garde pas comme morts ou muets ainsi que des tombeaux, à visiter avec nostalgie et recueilli, mais bel et bien que la conscience d'être s'est faite d'eux, de ce seul matériau, et qu'ils en restent la substance et l'infini, la conscience d'être et même le sens moral

qui affranchit ainsi, ici, de les visiter, et tant mieux, de les évoquer, encore mieux, à l'avenir il n'y aura plus, ce chemin cette fragilité probable à ce lieu du corps qu'est

le passé,

cette atteinte possible, lors refusée

tout est passé par là de nombreuses fois, par ce chemin de chèvre brouillé des tiges d'églantier, comme à bien d'autres endroits que surtout je ne chercherai pas à invoquer ici laissons, la conscience ne faire que flotter à la surface des choses et d'elle-même, suivant le sens de l'oubli et des jeux d'ombre - non pas délibérés ni même agis, mais venus de l'ombre toute, de son pouvoir meuble, mouvant, d'oblitérer, tout à tour, des zones sécables, et séquentielles, de son pouvoir de doute -

une fois se fut dite, franchit le fleuve

qu'il reste à être moins gratuit, à savoir ce qu'est ensuite mériter d'exister, laisser couler, cette encre du doute, à tous 
ces canaux de la pensée qui n'aident pas mais cèdent, là

comme cède, quand on s'y adosse, une forme liquide mais qui n'est plus d'heure, ici, on le dit, une fois le fleuve franchi

liquidité de l'heure et de la forme mémoire, vers son aboli, souhaitable, souhaité, comme un projet, à tenir la distance c'est dire, et la garder et la mener, le plus loin possible

la mémoire sombre

pouvant être quelque chose comme un effet de texte une écriture avide, ce ne peut être autre, déjà, une calamité ou, autant, une constellation impure, de signes anonymes ou pas même - la désignation ne cède, pourvu que cela n'aide, droit devant, ni, ne cèle, s'agissant de taire et non de protéger -

ce pouvait être quelque chose comme un effet de texte comme un effet de tête, de mémoire ou de caprice tel, soudain une emportée trop franche l'espoir du franchi le thème d'une transgression en son texte, ou ce dire avec une graphie têtue ici, affirmée comme un titre, espérée agissante dans son instance, le dit retour, qui s'obstine à ne rien franchir mais à déclarer franchi

à évacuer, successivement, et s'acharner à se sentir, toujours, en prise, des indices ostensibles de perte, pourtant, une souplesse excessive de la forme qui va, ainsi, jusqu'à la rupture, rigoureusement illisible,

la surface en attestait la trace des remous internes, répercutés, se dissout, au-dessous, perdant son effet de glace ou de miroir en tout cas d'écran et d'aplomb

la surface se met à laisser surgir le fond et se troubler, la surface en détestait l'aspect accidentel, la trivialité opposée à cet impeccable vertical qu'elle opposait à tout, à toute image, jusqu'alors, toujours en représentation -

or, le pire n'est jamais que le contraire du bon signe, juste, renier tout espoir dans l'indice d'une justice.

s'employer à, dessiner comme contours - destinés à les détours d'un texte illisible comme son désir, son c'est-à-dire propre, ce désir impossible à investir, 
qui n'en cesse pas moins d'être et de s'en ressentir le contour de l'écrire devrait être de même, absolu, absolue réponse impossible à ce désir contradictoire contredit mille fois et irritant d'obscur, de refus, de refuges, de fuites, de redites ou de poursuites...

si les contours s'en forment, c'est qu'il y a une chair, et ce, malgré l'étirement extrême du silence ou des nerfs

malgré les postures et les retraits successifs, sentir refluer du monde les apparences et les forfaits, cet univers du double et du pire, où le moindre style préfêre son écho en stylo, ce, plutôt qu'en stylet, si tant est de laisser trace ou pénétrer, juste en dessous de la surface sans la moindre idée du profond juste, à nouveau, mettre les pieds au fond, sous le niveau, toujours sans poser la question, de ce qui peut se passer, juste comme on éliminerait, les apparences et les reflets ici, qui rassureraient comme ils ont toujours rassuré, assuraient le monde dans son image en ne le renvoyant pas autre que tout, tout surface et superficie, l'espace d'une image et son retour

sur ce concentré d'image qu'est la surface réfléchissante tout est lisse et transgressible comme une envie de trouver matière fouiller, laisser se déposer ou, se précipiter, l'immédiat, et trouer la surface ou la chair pénétrer enfin un corps qui sait

la surface du fleuve franchi, à franchir à son tour?

alors que, son corps, seul, le vôtre c'est dire, est un corps qui sait, qui contient ou comprend, comprendrait, à sa façon, de fuir, d'esquiver - seuls vos silences font réponse, étendus à la façon d'un espace, où être à la fois exposé et confiant -

où surtout n'être pas, ce qui en est le plus fort attrait toujours désiré en votre absence, de si rares fois de face que c'en est troublant, à ces moments où parole ne masque, ne détourne de cette surprise, et le silence laisse ainsi, sans voile et sans voix, offre à la pensée cette naissance improbable très hautement, d'un instant où parole cèderait cesserait de céler, laisserait se vivre et être vive, vivante, de chair - laisserait place aux deux êtres -

sans que cela soit même un vœu, juste une forme de vertige, pour rapide qu'il soit - un autre vertige naissant, naquit, de cette persévérance profonde, à tenir la ligne ici définie 
parole de fleuve encore, cette seule muette montée ce silence gonflé que rien ne sait encore endiguer la façon dont l'eau gagne, longuement, menaçante, et lente, à en sentir mieux la puissance, au fil, de l'eau et des jours, avant de desserrer ensuite, la prise, l'emprise des flots, de lâcher les prés qui avaient été conquis, inondés du grand silence, et puis abandonnés désertés presque vite, et vides de cette présence forte et naturelle, et précise, qui se nourrit d'elle-même et en excède son corps

et si le fleuve est franchi, il isole plus encore, une fois pris dans le cercle des hautes eaux, les réfugiés des rives abolies -

sous les fenêtres les eaux ont atteint les départs des branches au-delà des troncs au-delà des bords fluctuants, quelques îlots effleurent l'air encore aux limites des champs, se manifestent, dans limpeccable victoire de la surface miroir, grise surtout, qui rend au décor son indifférenciation première du petit matin avant que le volume de la clarté ne le fixe bien immuable - ici libéré soudain de cette prescription, reprenant l'indéterminé, l'influence du ciel, le modelé des eaux redistribuent les rôles les forment, dans une plus parfaite unité - non plus le partage des eaux, mais le passager ressaisissement du décor en un corps 